\title{
Experimental Validation of a Real-Time Energy Management System Using Multi-Period Gravitational Search Algorithm for Microgrids in Islanded Mode
}

\author{
Mousa Marzband ${ }^{\mathrm{a}, \mathrm{b}}$, Majid Ghadimi ${ }^{\mathrm{b}}$, Andreas Sumper, ${ }^{\mathrm{d}, \mathrm{e}}$, José Luis Domínguez-García ${ }^{\mathrm{c}}$ \\ ${ }^{a}$ The University of Manchester, School of Electrical and Electronic Engineering, Ferranti Building, Manchester, M13 9PL, UK \\ ${ }^{b}$ Department of Electrical Engineering, Lahijan Branch, Islamic Azad University, Lahijan, Guilan, Iran \\ ${ }^{c}$ Catalonia Institute for Energy Research (IREC), Jardins de les Dones de Negre 1, 08930 Sant Adrià de Besòs, Barcelona, Spain \\ dDepartament d' Enginyeria Eléctrica, EU d'Enginyeria Técnica Industrial de Barcelona (CITCEA-UPC), Universitat Politècnica de Catalunya (UPC), C. Comte \\ d'Urgell, 187, Pl. 2. 08036 Barcelona, Spain \\ ${ }^{e}$ Centre d'Innovació Tecnològica en Convertidors Estàtics i Accionaments (CITCEA-UPC), Departament d'Enginyeria Elèctrica, Universitat Politècnica de \\ Catalunya, ETS d'Enginyeria Industrial de Barcelona, Av. Diagonal, 647, Pl. 2. 08028 Barcelona, Spain
}

\begin{abstract}
Both performance optimization and scheduling of the distributed generation (DG) are relevant implementing an energy management system (EMS) within Microgrid (MG). Furthermore, optimization methods need to be applied to achieve maximum efficiency, improve economic dispatch as well as acquiring the best performance. This paper proposes an optimization method based on gravitational search algorithm to solve such problem in a MG including different types of DG units with particular attention to the technical constraints. This algorithm includes the implementation of some variation in load consumption model considering accessibility to the energy storage (ES) and demand response (DR). The proposed method is validated experimentally. Obtained results show the improved performance of the proposed algorithm in the isolated MG, in comparison with conventional EMS. Moreover, this algorithm which is feasible from computational viewpoint, has many advantages as peak consumption reduction, electricity generation cost minimization among other.
\end{abstract}

Keywords: Day Ahead Scheduling, Demand Response, Energy Management System, Microgrid, Optimal Operation and Scheduling, Gravitational Search Algorithm.

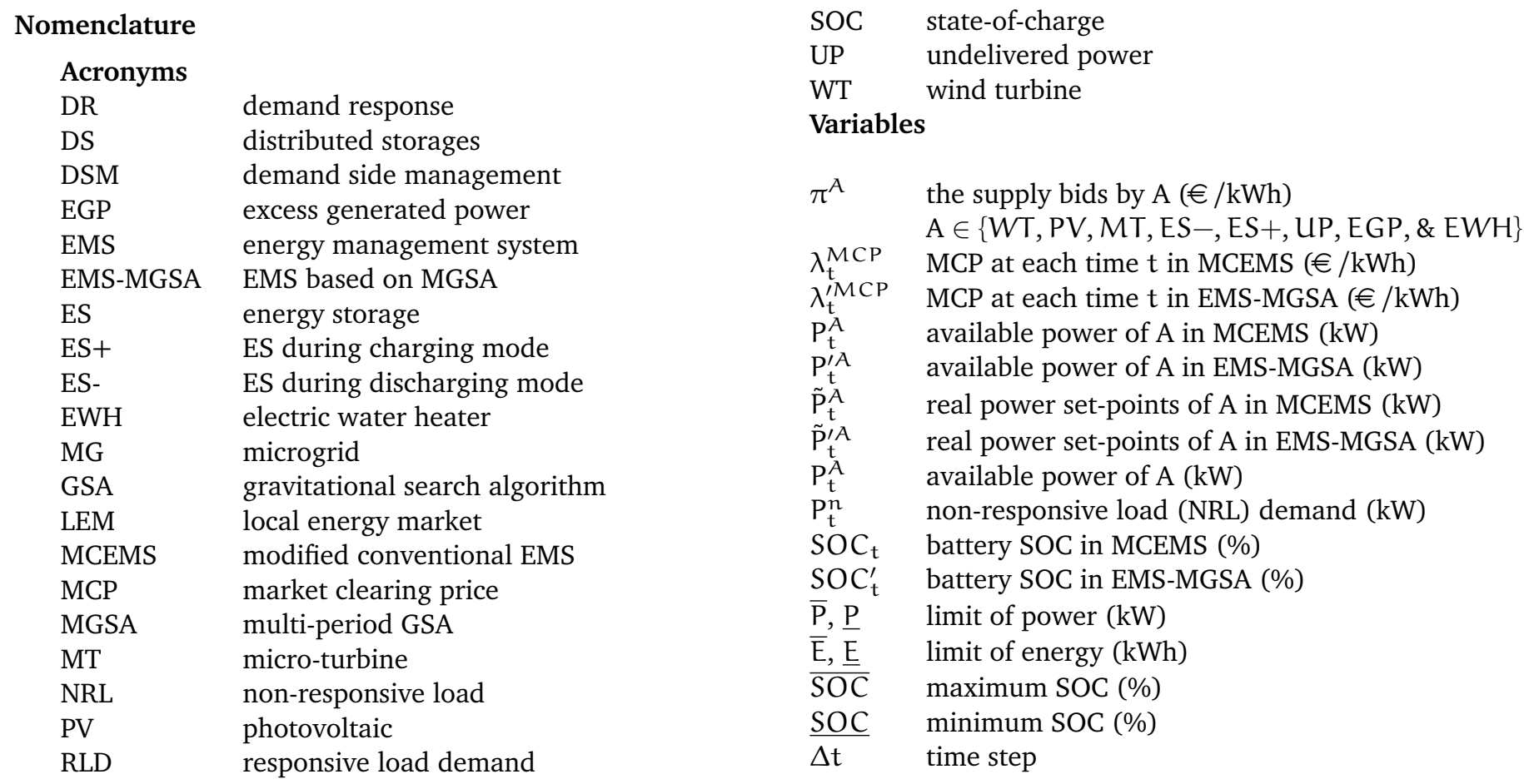

Email address: mousa.marzband@manchester.ac.uk, Tel. $+44(0) 1613064654$, Fax.
author (Mousa Marzband) 


\section{Introduction}

Distributed generation can become integrated into distribution power systems through a controllable platform called Microgrid (MG) which includes converter-based systems [1]. In the MGs, if energy generation sources are not enough to feed the requested load, the system is not able to match supply demand. To apply a proper energy management system (EMS) is crucial to avoid this problem. An EMS makes possible the optimum implement and use of distributed energy sources. Failing of these systems in load feeding is possible if the total demand is more than the maximum capacity of the generation sources.

Applying either supporting systems such as diesel generators, distributed storages (DS) or implementing demand side management (DSM), may be useful to reduce a supplydemand mismatch [2-4].

Special attention is considered in using support systems as DSM and storage systems in this paper. The main objectives of DSM program is minimizing mismatch between feed power and load during consumption peak by changing the system load curve. The variation of system load curve can be done through both the distribution system facilities and enduse customers [5-7]. Demand response (DR) is a mechanism in which consumers participate voluntarily in reducing consumption peak by changing the consumption model. Consumers participating in DR receive some energy cost benefit [8]. Large scale participation in DR into distribution systems can be managed by applying aggregators. The role of an aggregator is gathering all the DRs requested by the end users, to present them in wholesale electricity market $[9,10]$. One of the key goals of DR management plans is shift power demand to nonpeak hours [8]. The combined operation of DS and DR brings more reliability into distribution system operation [11]. DS may include some constant storage systems (e.g. battery energy storage) and mobile storage (e.g. plugin electric vehicle). On the other hand, the DR can also be treated as a load shaping tool in distribution grids with high penetration of plug-in loads, such as electric vehicle [12]. Applying DR in smart distribution networks with several micro sources requires a complex and fast EMS [13-15]. Thus, optimal techniques are required to fulfill the aforementioned cases.

The use of deterministic methods is not complex and time consuming to solve optimum problems with large dimensions. Hence, these problems can be solved with the non-deterministic polynomial-hard (NP-hard) problem [16]. There is increased tendency for using population algorithms in recent years. These are inspired by social and natural behaviors. Several research is done over these algorithms dealing with solving complex numerical problems. Various innovative methods are introduced for solving optimization problems like genetic algorithm (GA) [17, 18], simulated annealing (SA) [19], ant colony optimization (ACO) [20] and particle swarm optimization (PSO) [21-23].

For that reason, a swarm intelligence method called multiperiod gravitational search algorithm (MGSA) is applied in this paper. Its main benefits include exploration operation can be started in several working point at the same time [24], without memory and evaluation of the masses can be done in each interval [25], multi-agent considering an agent can be evaluated by noting the total force obtained by all of the other agents [26], update results applying the quality of solving the problem with attention to fitness function [27]. Because of this, it is implemented for EMS.

This paper aims to introduce and validate experimentally an EMS based on MGSA within MG. The main contribution are as follows:

1. The implementation of MGSA algorithm for using in MG applications with the following characteristic:

(a) Presented method for solving problems with $\mathrm{K}$ spaces $\mathrm{N}$ dimensional; It is useful for calculation of each interval.

(b) Modification of the relationship of velocity, displacement and force for compatibility with MG applications.

2. Experimental implementation of the MGSA for EMS which demonstrates that it is fast, extendible and flexible;

\section{Problem formulation}

The system under analysis encompasses a stand-alone wind turbine (WT), photovoltaic (PV), microturbine (MT), and energy storage (ES) system.

\subsection{The mathematical implementation of MGSA units}

The following assumptions will be considered for the optimization problem:

- The voltage level in all of the points of MG is the same;

- The power loss is neglected;

- The reactive power flow is neglected.

The optimization problem is defined according to the following objective function:

$$
\min \sum_{t=1}^{m}\left(\mathbb{C}_{t}^{g}+\mathbb{C}_{t}^{\prime g}+\mathbb{C}_{t}^{E S-}-\mathbb{C}_{t}^{\ell}-\mathbb{C}_{t}^{E S+}+\Omega_{t}\right) \times \Delta t
$$

where $m$ is the number of time periods in the scheduling time horizon $\mathrm{T}, \mathbb{C}_{\mathrm{t}}^{\mathrm{g}}, \mathbb{C}_{\mathrm{t}}^{\prime \mathrm{g}}$ depict to the cost of energy produced by renewable and non-renewable generation units in period $t, \mathbb{C}_{t}^{E S+}, \mathbb{C}_{t}^{E S-}$ present the cost of energy produced by $E S$ units during charging and discharging operation mode in period $t, \mathbb{C}_{t}^{\ell}$ is the cost of energy consumed by responsive load demand (RLD) and $\Omega_{t}$ is the penalty cost resulting from undelivered power (UP) during the time period $t$. 
The production cost of different unit types can be determined as

$$
\begin{aligned}
& \mathbb{C}_{\mathrm{t}}^{\mathrm{g}}=\sum_{\mathrm{k}=1}^{\mathrm{n}^{\mathrm{g}}} \pi_{\mathrm{t}}^{\mathrm{k}, \mathrm{g}} \cdot \mathrm{P}_{\mathrm{t}}^{\mathrm{k}, \mathrm{g}} \\
& \mathbb{C}_{\mathrm{t}}^{\prime \mathrm{g}}=\sum_{\mathrm{k}=1}^{\mathrm{n}^{\prime g}} \pi_{\mathrm{t}}^{\prime \mathrm{k}, \mathrm{g}} \cdot \mathrm{P}_{\mathrm{t}}^{\prime \mathrm{k}, \mathrm{g}} \\
& \mathbb{C}_{\mathrm{t}}^{\ell}=\sum_{\mathrm{k}=1}^{\mathrm{n}^{\ell}} \pi_{\mathrm{t}}^{\mathrm{k}, \ell} \cdot \mathrm{P}_{\mathrm{t}}^{\mathrm{k}, \ell} \\
& \mathbb{C}_{t}^{E S}+\sum_{k=1}^{n E S} \pi_{t}^{k, E S+} \cdot X_{t}^{E S} \cdot P_{t}^{k, E S+} \\
& \mathbb{C}_{t}^{E S-}=\sum_{k=1}^{n} \pi_{t}^{k, E S-} \cdot\left(1-X_{t}^{E S}\right) \cdot P_{t}^{k, E S-} \\
& \Omega_{t}=\pi_{t}^{U P} \cdot P_{t}^{U P}
\end{aligned}
$$

where $\pi_{t}^{k, g}, \pi_{t}^{\prime k, g}$ represent the offer prices by the $\mathrm{k}^{\mathrm{th}}$ renewable and non-renewable resources during $t$ period, $P_{t}^{k, g}, P_{t}^{\prime k, g}$ are power output of $k^{t h}$ renewable and nonrenewable units during $t$ period, $n^{9}, n^{\prime g}$ indicate the number of renewable and non-renewable generation units installed in the MG system, $\Delta t$ is duration of $t$ period, $\pi_{t}^{k, \ell}$ is relevant to offer price by the $k^{\text {th }}$ RLD during $t$ period, $P_{t}^{k, \ell}$ is the consumed power by the $k^{\text {th }}$ RLD during $t$ period, $\pi_{t}^{\mathrm{UP}}$ is the offer price when the system has UP and $P_{t}^{U P}$ is the amount of power that has not been supplied by MG.

The total generation cost should be minimized subject to the following constraints.

- Power balance

$$
\begin{aligned}
& \sum_{k=1}^{n^{g}} P_{t}^{k, g}+\sum_{k=1}^{n^{\prime g}} P_{t}^{\prime k, g}+\sum_{k=1}^{n^{E S}}\left(1-X_{t}^{E S}\right) \cdot P_{t}^{k, E S-}+P_{t}^{U P}= \\
& P_{t}^{N R L}+\sum_{k=1}^{n^{\ell}} P_{t}^{k, \ell}+\sum_{k=1}^{n^{E S}} X_{t}^{E S} \cdot P_{t}^{k, E S+}
\end{aligned}
$$

- The renewable generation unit [28]

$$
0 \leqslant \sum_{k=1}^{n^{g}} P_{t}^{k, g} \leqslant \bar{P}^{g}
$$

where $\bar{P}_{t}^{g}$ is the maximum available power by the renewable resources at time $t$.

- The non-renewable generation units [20, 28]
- Capacity limits

$$
X_{t}^{i, g} \cdot \underline{P}^{\prime i, g} \leqslant P_{t}^{\prime i, g} \leqslant X_{t}^{g} \cdot \bar{P}^{\prime i, g}, X_{t}^{g} \in\{0,1\}
$$

where $\underline{P}^{i, g}$ and $\bar{P}^{\prime i, g}$ are the minimum and the maximum power outputs of the $i^{\text {th }}$ non-renewable generation unit in $\mathrm{kW}$.

- Maximum and minimum operating times [20, 29]

$$
\left[T_{t-1, O N}^{\prime i, g}-\bar{T}^{i, g}\right] \cdot\left[X_{t-1}^{\prime i, g}-X_{t}^{\prime i, g}\right] \geqslant 0
$$

$$
\left[T_{t-1, \text { OFF }}^{\prime i, g}-\underline{T}^{\prime i, g}\right] \cdot\left[X_{t}^{\prime i, g}-X_{t-1}^{\prime i, g}\right] \geqslant 0
$$

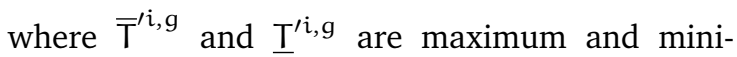
mum up and down time of $i^{\text {th }}$ non-renewable unit (min), respectively. $\mathrm{T}_{\mathrm{t}, \mathrm{ON}}^{\prime i, g}$ and $\mathrm{T}_{\mathrm{t}, \mathrm{OFF}}^{\prime i, g}$ represent time duration for which unit $\mathrm{i}$ have respectively been $\mathrm{ON}$ and $\mathrm{OFF}$ at time t. $\mathrm{X}_{\mathrm{t}-1}^{\prime i, g}$ is a binary variable indicating the commitment state of unit $i$ at time $t$ (i.e., $X_{t}^{\prime i, g}=1$ when the $i^{\text {th }}$ unit is on, and $X_{t}^{i, g}=0$ when it is in off state).

- Ramp-rate limit

$$
\begin{aligned}
& \left(P_{t}^{\prime i, g}-P_{t-1}^{\prime i, g}\right) \leqslant R U^{i} \\
& \left(P_{t-1}^{\prime i, g}-P_{t}^{\prime i, g}\right) \leqslant R D^{i}
\end{aligned}
$$

where $\mathrm{RU}^{\mathrm{i}}$ and $\mathrm{RD}^{\mathrm{i}}$ are ramp up and down of unit $i(\mathrm{~kW} / \mathrm{min})$, respectively. $t$ is the current time interval (min).

- ES constraints [20, 30, 31]

- Energy storage limits

$$
\mathrm{E}_{\mathrm{t}}^{\prime \mathrm{ES}} \leqslant \overline{\mathrm{E}}^{\mathrm{ES}}
$$

- Maximum discharge limit

$$
\begin{aligned}
& \left(1-X_{t}^{E S}\right) \cdot P_{t}^{E S-} \leqslant \bar{P}^{E S-}, P_{t}^{E S-} \geqslant 0 \\
& X_{t}^{E S} \in\{0,1\}
\end{aligned}
$$

- Maximum charge limit

$$
X_{t}^{E S} \cdot P_{t}^{E S+} \leqslant \bar{P}^{E S+}, P_{t}^{E S+} \geqslant 0
$$

Eq.(16) shows that when the ES is in the discharging mode (i.e., $X_{t}^{E S}=0$ ), the discharging power cannot exceed the maximum discharging power of the ES. The same operation characteristic is defined for the charging mode, which is given in Eq.(17). 
- Maximum discharge limit considering the stored energy

$$
\left(\left(1-X_{t}^{E S}\right) \cdot P_{t}^{E S-} \cdot \Delta t\right) \leqslant E_{t-1}^{\prime E S}
$$

- Maximum charge limit considering the stored energy

$$
\left(\left(X_{t}^{E S} \cdot P_{t}^{E S+} \cdot \Delta t\right)+E_{t-1}^{E S}\right) \leqslant \bar{E}^{E S}
$$

Eq.(18) simply ensures that the discharged energy will not exceed the total available energy in the ES. Also, total charging energy and stored energy up to time $t$ cannot exceed the maximum energy stored in the ES. Both conditions are modeled in Eq.(19).

- Energy balance in ES

$$
E_{t}^{E S}=E_{t-1}^{E S}+\left(X_{t}^{E S} \cdot P_{t-1}^{E S+}-\left(1-X_{t}^{E S}\right) \cdot P_{t-1}^{E S-}\right) \times \Delta t
$$

- Battery SOC

$$
\mathrm{SOC}_{\mathrm{t}}=\frac{\mathrm{E}_{\mathrm{t}}^{\mathrm{ES}}}{\mathrm{E}_{\mathrm{Tot}}^{\mathrm{ES}}}
$$

- ES limit

$$
\underline{E}^{E S} \leqslant E_{t}^{E S} \leqslant \bar{E}^{E S}
$$

- Demand response constraints

- DR power limit

$$
\sum_{t} P_{t}^{D R}=\sum_{t} P_{t}^{U P}
$$

The RLD loads are also considered with Eq.(23), where the total controllable load demand should always be equal the pre-defined UP during a daily operation system. This is because it is desired to store excess generation power in the ES.

- Electric water heater (EWH) constraint

$$
P_{t}^{\prime E W H} \leqslant \bar{P}^{E W H}
$$

The total excess generated power (EGP) by generation and storage devices should be used to supply DR, ES and EWH during daily system operation. This case can be guaranted by

$$
\begin{aligned}
& P_{t}^{\prime E G P}=X_{t}^{E S} \cdot P_{t}^{E S+}+X_{t}^{D R} \cdot P_{t}^{\prime D R}+P_{t}^{\prime E W H} \\
& X_{t}^{D R} \in\{0,1\}
\end{aligned}
$$

where $X_{t}^{D R}$ is a binary variable for DR status (i.e. 1 if the request is in service and 0 otherwise).

$$
\sum_{t} P_{t}^{E G P}=\sum_{t} X_{t}^{E S} \cdot P_{t}^{E S+}+\sum_{t} P_{t}^{D R}+\sum_{t} P_{t}^{E W H}
$$

In addition, the summation of consumed power by these customers should be equal to the summation of EGP during a daily operation system as shown mathematically in Eq. (26).

\subsection{Implementation of the EMS based on GSA}

\subsubsection{Introduction to the MGSA Method}

In this algorithm, the search of optimum points is done based on gravitational Newtons' laws governing the dynamics of the masses [24, 32-34]. In this method, $N$ masses are considered and each mass is placed in the $\mathrm{D}$ dimensional space. The position of each mass is an answer of the problem.

${ }^{d} F_{t}^{i},{ }^{d} a_{t}^{i}$ and ${ }^{d} X^{i}$ are calculated according to forces between masses and the gravitational forces of other masses. Where ${ }^{d} a_{t}^{i}$ are ${ }^{d} X^{i}$ the acceleration of motion in the $d^{t h}$ dimension and the new position of the masses. Then, the evaluation of the masses is done based on the objective function. The heavier masses have higher fitness values; they depict good optimal solution to the problem and they move slowly than lighter ones representing worse solutions.

This process continues until convergence of the results and/ or fulfillment of stopping condition. Considering a system with $\mathrm{N}$ masses, the position of the $i^{\text {th }}$ mass in the $D^{\text {th }}$ dimensional space is defined as

$$
X=\left[{ }^{1} X^{1}, \ldots,{ }^{d} X^{i}, \ldots,{ }^{D} X^{N}\right]
$$

where ${ }^{d} X^{i}$ presents the position of $i^{\text {th }}$ mass in the $d^{\text {th }}$ dimension. The force exerted on mass $i$ from mass $j$ in the direction of dimension $d$ at the time $t$ (iteration $t$ ) is defined as

$$
{ }^{d} F_{t}^{j, i}=G_{t} \frac{M_{t}^{i} \cdot M_{j}}{R_{t}^{j, i}+\varepsilon}\left({ }^{d} X_{t}^{j}-{ }^{d} X_{t}^{i}\right), j \neq i
$$

where $M_{t}^{i}$ and $M_{t}^{j}$ are masses of body $i$ and $j$, respectively. $R_{t}^{j, i}$ is distance between $i$ and $j$ at $t^{\text {th }}$ repetition and $\varepsilon$ is an extremely small constant to avoid division by zero. $R_{t}^{j, i}$ can be achieved as

$$
R_{t}^{j, i}=\sqrt{\sum_{d=1}^{D}\left({ }^{d} X_{t}^{j}-{ }^{d} X_{t}^{i}\right)^{2}}
$$

The resultant of the forces applied on $i^{\text {th }}$ mass in the $d^{\text {th }}$ dimension is calculated by

$$
{ }^{d} F_{t}^{i}=\sum_{j=1, j \neq i}^{N} \rho^{f} \cdot d F_{t}^{j, i}
$$

where $\rho^{f}$ is a random number between zero and one. The resulting response becomes farther than the optimum response because of using the random places which is less affected by larger masses. To avoid unexpected results, Eq. (30) is modified as 


$$
{ }^{d} F_{t}^{i}=\sum_{j=1, j \neq i}^{N}{ }^{d} F_{t}^{j, i}
$$

The force exerted on $i^{\text {th }}$ mass creates acceleration in the direction of $\mathrm{d}^{\text {th }}$ dimension as

$$
\mathrm{d}_{\mathrm{t}}^{\mathrm{i}}=\frac{\mathrm{d}_{\mathrm{t}}^{\mathrm{i}}}{M_{\mathrm{t}}^{\mathrm{i}}}
$$

New velocity $\left({ }^{d} V_{t+1}^{i}\right)$ and relocation $\left({ }^{d} X_{t+1}^{i}\right)$ mass $i$ in the direction of dimension $d\left({ }^{d} X_{t+1}^{i}\right)$ can be calculated as

$$
\begin{aligned}
& { }^{d} V_{t+1}^{i}=\rho^{v} \cdot{ }^{d} V_{t}^{i}+{ }^{d} a_{t}^{i} \times T \\
& { }^{d} X_{t+1}^{i}={ }^{d} X_{t}^{i}+{ }^{d} V_{t+1}^{i} \times T
\end{aligned}
$$

where $\rho^{v}$ is a random number between zero and one.

In the velocity relation, using random function (i.e. $\rho^{v}$ ) causes the increase of the exploration of the algorithm. In these relations, the time duration parameter of the motion (i.e. T) is considered 1 . In the modified algorithm, the control parameter $T$ is considered as the time duration of the $i^{\text {th }}$ mass from the present position to the next position. According to the done analysis, it is indicated by the trial-and-error hypothesis that the value of 2 seconds leads to a better answer.

The gravitational constant $\mathrm{G}$ is obtained by

$$
\mathrm{G}_{\mathrm{t}}=\mathrm{G}_{0} \times \exp \left(-\alpha \frac{\mathrm{t}}{\overline{\mathrm{I}}}\right)
$$

where $G_{0}$ is the initial value of the gravitational constant, $\alpha$ is the controlling parameter, $\mathrm{t}$ is the current iteration and $\overline{\mathrm{I}}$ is the total number of iterations. After each iteration and movement, the bodies must be evaluated based on the objective function in their new positions. In this order, the masses with better position (smaller objective function) have heavier mass and members with worse position have lighter mass. The masses can be computed as

$$
M_{t}^{i}=\frac{f i t_{t}^{i}-\min \left(f i t_{t}^{i}\right)}{\max \left(f i t_{t}^{i}\right)-\min \left(f i t_{t}^{i}\right)}
$$

where $\mathrm{fit}_{t}^{i}$ is the value of the objective function of mass $i$ in the $t^{\text {th }}$ iteration, $\max \left(f i t_{t}^{i}\right)$ and $\min \left(f_{i t}^{i}\right)$ are respectively the worst and the best value of the objective function in the $\mathrm{t}^{\text {th }}$ iteration.

One way to perform a good compromise between exploration and exploitation is to reduce the number of masses with lapse of time in Eq. (30). After several iterations only the best masses are chosen for calculating the gravity force exerted on other masses.

For example, at the beginning of the process, all the masses participate in the gravity force inside local optimum points. But, this number reduces in next iterations. In order to reach this goal, the percentage of members is defined as a control parameter $\xi$ at the end of time. $\Psi_{t}$ is defined as the best of the masses at each iteration. $\Psi_{t}$ can be estimated as

$$
\Psi_{\mathrm{t}}=\operatorname{round}\left(\left[\xi+1-\frac{\mathrm{t}}{\overline{\overline{\mathrm{I}}}} \times(100-\xi)\right] \times \mathrm{N}\right)
$$

On this basis, the resultant of the forces exerted on mass $i$ in the dimension $d$ is calculated as Eq.(31) where $j$ exists on $\Psi_{\mathrm{t}}$.

\subsubsection{MGSA application to MG case}

It is noteworthy that one day period is equal a total space of $48 \times \mathrm{D}$ dimensions. $\mathrm{D}$ is the number of independent parameters. Space and the number of variables must be failed in the algorithm at the first moment.

Independent variables in the matrices $\overline{X M}_{j}$ for mass $j$ is defined as follows:

$$
\overline{\mathrm{XM}}_{j}=\left[\overrightarrow{\mathrm{P}}_{j}^{W T}, \overrightarrow{\mathrm{P}}_{j}^{\mathrm{PV}}, \overrightarrow{\mathrm{P}}_{j}^{M T}, \overrightarrow{\mathrm{E}}_{j}^{E S}, \overrightarrow{\mathrm{P}}_{j}^{\mathrm{DR}}\right]
$$

The variables $\vec{P}_{j}^{W T}, \vec{P}_{j}^{P V}, \vec{P}_{j}^{M T}, \vec{E}_{j}^{E S}$ and $\vec{P}_{j}^{D R}$ are respectively the vector of the powers of WT, PV, MT, ES and DR power. MGSA method considers a space with the dimension $\mathrm{nT} \times 5 \times \mathrm{N}$, where, $\mathrm{nT}$ represent the number of the periods, 5 is the number of independent variables and $N$ is the number of masses. The rest of the existing variables are dependent on these previous 5 . The position of the masses are determined by the matrices $\overline{X M}_{j}$. The forces between the masses affect this variables directly. By knowing $\vec{E}_{j}^{E S}$ at different times of a period, the parameters $\vec{P}_{j}^{E S}+$ and $\vec{P}_{j}^{E S}-$ can be derived. Having the generated and consumed powers, the parameters $\vec{P}_{j}^{E W H}$ and $\vec{P}_{j}^{U P}$ can be obtained through power balance. For this purpose, two matrix variables namely EM and $\mathrm{YM}$ are defined as

$$
\begin{gathered}
\overline{\mathrm{EM}}_{j}=\left[\overrightarrow{\mathrm{P}}_{j}^{\mathrm{ES}+}, \overrightarrow{\mathrm{P}}_{j}^{\mathrm{ES}-}, \overrightarrow{\mathrm{X}}_{j}^{\mathrm{ES}}\right] \\
\overline{\mathrm{YM}}_{j}=\left[\overrightarrow{\mathrm{P}}_{j}^{\mathrm{UP}}, \overrightarrow{\mathrm{P}}_{j}^{\mathrm{E} W \mathrm{H}}\right]
\end{gathered}
$$

In this order, the variables $\overline{\mathrm{EM}}_{j}$ and $\overline{\mathrm{YM}}_{j}$ are calculated indirectly. It should be noted that all the independent and dependent variables take part in the calculation of objective function. As a result, the effect of gravitational forces is directly applied over $\overline{X M}_{j} . \overline{E M}_{j}$ and $\overline{Y M}_{j}$.

Figure 1 shows an example scheme. Total response can be achieved from the combination of optimum responses obtained in similar spaces. The number of $\mathrm{N}$ masses are considered in each space. Optimum point can be determined by noting the cost function and the technical constraints considered. The initial SOC in each space is equal to the last condition of the ES in the previous space. As illustrated in this figure, an increase in the distance between two masses means decreasing the gravity force between them. Every mass accelerate toward the result force acting on it from the other masses. In this figure, $F_{t}^{i, j}$ is the force that exerts on $M_{t}^{i}$ from $M_{t}^{i}$. $F_{t}^{1}$ produces the overall net force applied to $M_{t}^{1}$ mass. 


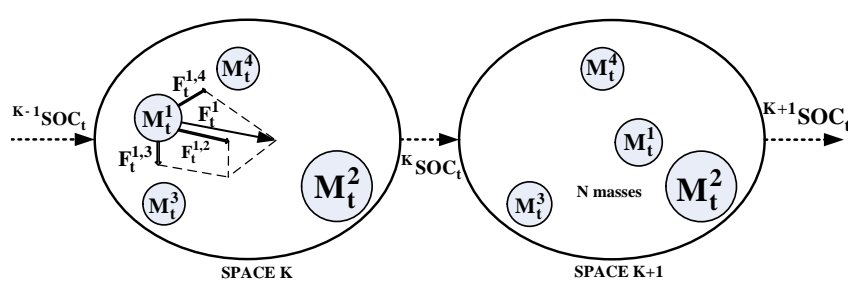

Figure 1: Scheme of masses displacement

\subsection{Mathematical implementation of the local energy market (LEM) unit}

In this unit, the single sided auction structure is used to find the value of MCP in each time interval. In this unit, the energy consumed and the energy generated are sent to the LEM unit. The algorithm used is presented in the previous papers [1].

\section{The algorithm suggested for MGSA}

EMS-MGSA algorithm is made up of two units including MGSA and LEM units as shown in Figure 2. The implementation of each units are explained in the following subsections.

\subsection{MGSA unit}

The flowchart of implementing this algorithm is shown in Figure 3. The stages of the process are briefly listed and related to the previous equation as follows:

1. identification of the space (Eq. (27));

2. the random initial value of the masses;

3. masses evaluation by calculating the values of the objective function;

4. updating the values of $M_{t}^{i}, \max \left(f i t_{t}^{i}\right), \min \left(f i t_{t}^{i}\right)$ and $\mathrm{G}_{\mathrm{t}}$ for all the masses (Eq. (35)-(36));

5. calculating the force resultant in different directions (Eqs. (30)-(35));

6. calculation of acceleration and velocity (Eq. (34) and (33));

7. updating the position of the masses (Eq. (34));

8. iteration of the stages 3 to 7 until the stopping conditions;

9. End

The Pseudo-code of MGSA unit is provided in Algorithm 1 for clarify. In addition, Algorithm 2 is a general outline of the proposed algorithm followed in detail by pseudo-code.
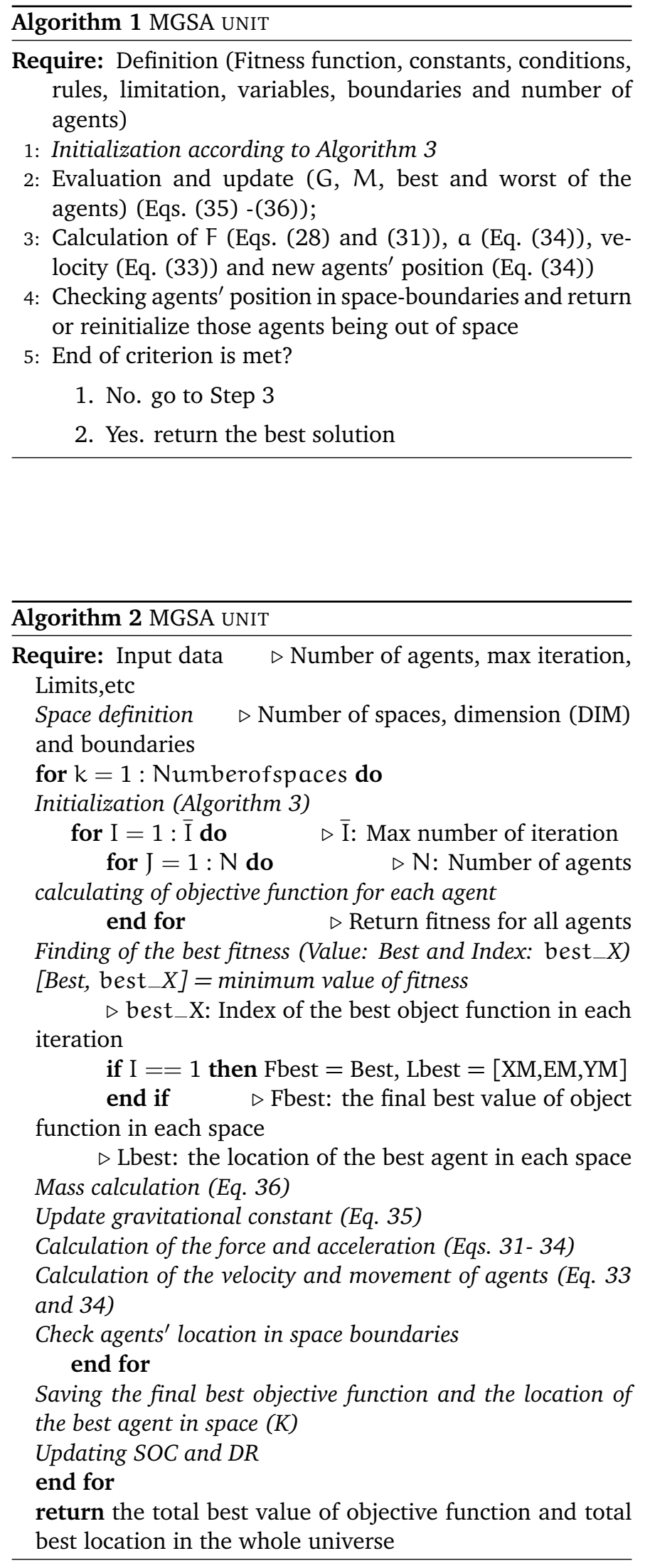

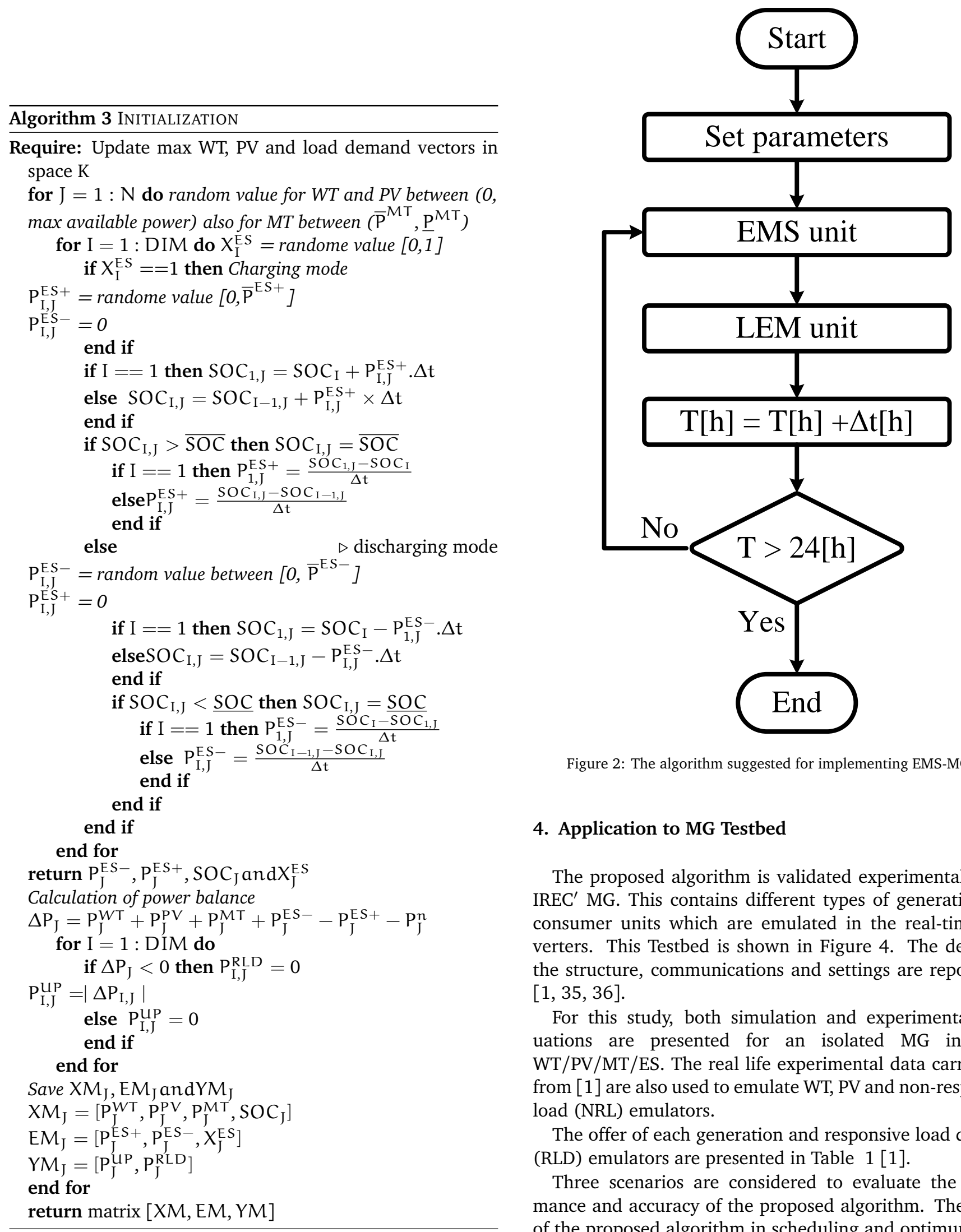

Figure 2: The algorithm suggested for implementing EMS-MGSA

\section{Application to MG Testbed}

The proposed algorithm is validated experimentally over IREC' MG. This contains different types of generation and consumer units which are emulated in the real-time converters. This Testbed is shown in Figure 4. The details of the structure, communications and settings are reported in $[1,35,36]$.

For this study, both simulation and experimental evaluations are presented for an isolated MG including WT/PV/MT/ES. The real life experimental data carried out from [1] are also used to emulate WT, PV and non-responsive load (NRL) emulators.

The offer of each generation and responsive load demand (RLD) emulators are presented in Table 1 [1].

Three scenarios are considered to evaluate the performance and accuracy of the proposed algorithm. The ability of the proposed algorithm in scheduling and optimum operation, minimizing the electricity generation cost and finally the generation side management are considered:

- Scenario $\sharp 1$ : Normal operation 


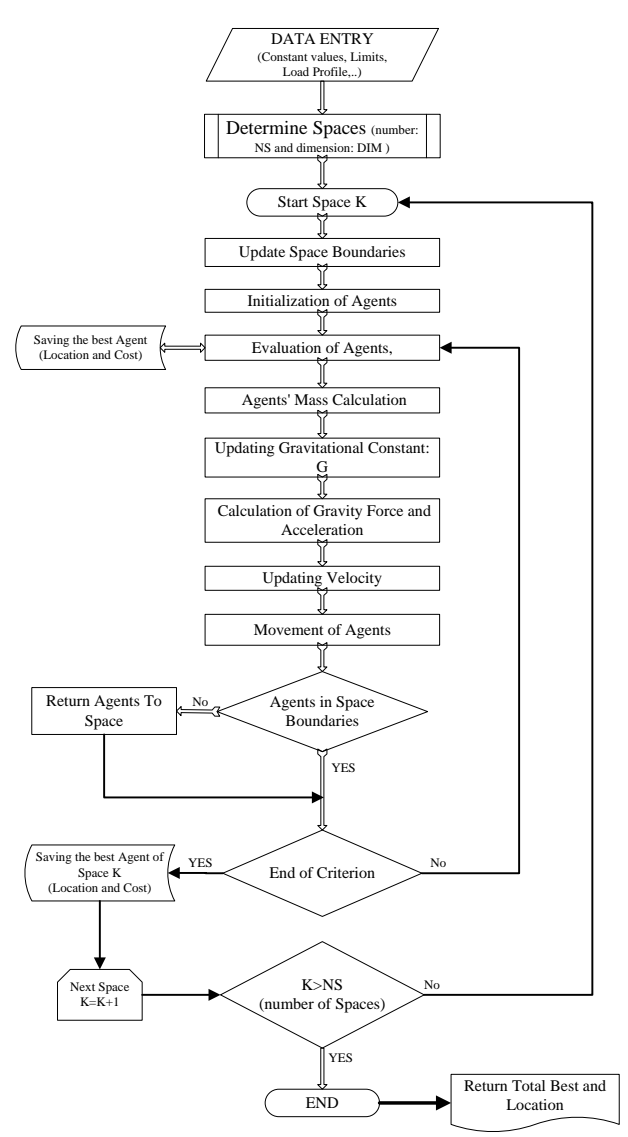

Figure 3: Graphical illustration of the process undertaken in the EMS unit

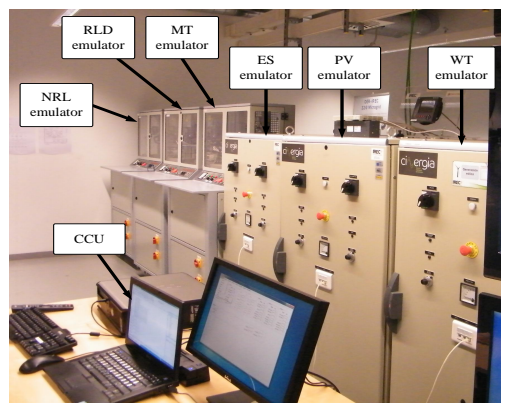

(a) IREC's MG

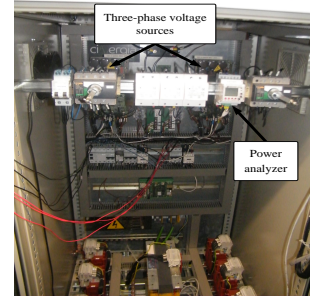

(b) Cabinet inside details
Figure 4: System configuration of IREC's MG Testbed

- Scenario $\sharp 2$ : Sudden load increase

- Scenario $\sharp 3$ : Plug and play ability

\section{Results and discussion}

This section describes the results of experimental evaluation under different scenarios.

SOC is shown in Figure 5 for the algorithms MCEMS and EMS-MGSA. At the first 6 hours of the system operation, ES in EMS-MGSA is always operated in charging mode. Nevertheless, in MCEMS, ES is mostly operated in discharging mode.
Table 1: The supply bids by the generation and consumers assets [€/kWh]

\begin{tabular}{|c|c|c|c|c|c|c|c|}
\hline$\pi^{W T}$ & $\pi^{\mathrm{PV}}$ & $\pi^{\mathrm{MT}}$ & $\pi^{\mathrm{ES}-}$ & $\pi^{\mathrm{ES}+}$ & $\pi^{\mathrm{UP}}$ & $\pi^{\mathrm{E} W \mathrm{H}}$ & $\pi^{\mathrm{DR}}$ \\
\hline 0.083 & 0.1 & 0.15 & 0.145 & 0.125 & 1.5 & 0.105 & 0.115 \\
\hline
\end{tabular}

This fact indicates that despite the MT offer is high, the optimization algorithm is decided to use MT for compensating the lack of power. This fact is shown in Figure 6(b). The algorithm during this period, in addition to supplying the load required power, produces the excess generated power (EGP) as shown in Figure 7(b), for feeding ES, EWH and DR. At the end of the second and third 6 hours of the system operation, ES system in both of the algorithms is almost completely discharged and SOC is approached almost to $\underline{\mathrm{SOC}}$. The key point is that at the fourth period of system operation, ES in the EMS-MGSA is started to operate in charging mode after a short period of discharging. As a result, the value of SOC in this algorithm reaches about $67 \%$ at the end of daily operation. While, its value is reached close to $\underline{\mathrm{SOC}}$ in the algorithm MCEMS, hence, ES definitely will show a better capability in the EMS-MGSA to support the system at the beginning of the next day.

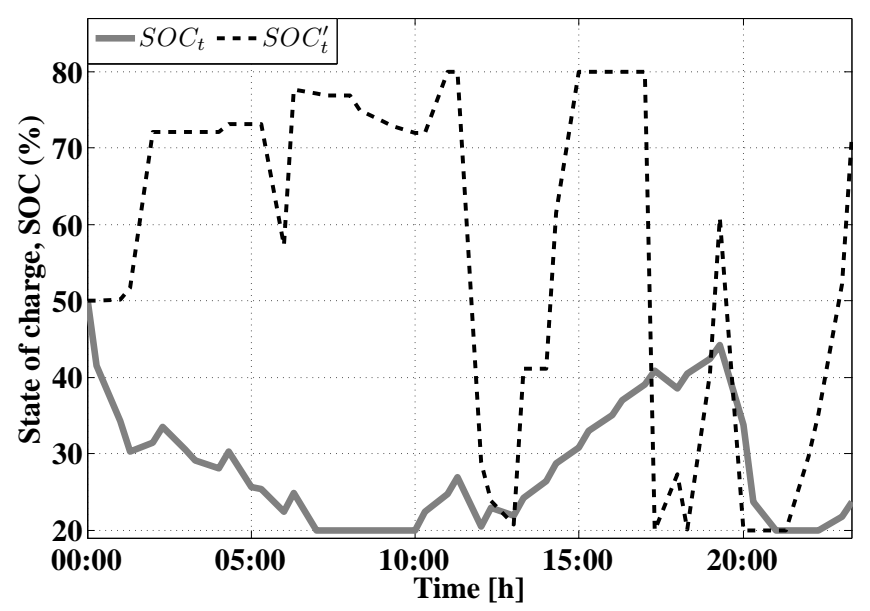

Figure 5: SOC during system operation (Solid light-gray line indicates MCEMS algorithm. Also, dash black lines represent output of EMS-MGSA algorithm)

ES and MT power profile during the MG daily operation are shown in Figures. 6(a) and 6(b), respectively. In the MCEMS, ES is operated about $42 \%$ period in charging mode, $29 \%$ in discharging mode and $29 \%$ in the idle mode during $24 \mathrm{~h}$ of system operation. However, in the EMS-MGSA algorithm, it is respectively operated 39.55 in the charging mode, $27 \%$ in the discharging mode and $33.5 \%$ in the idle mode. This shows that despite the higher offer of MT relative to ES, the EMS-MGSA algorithm uses MT in more time intervals. Noting that the minimum power generated by MT is equal to $\mathrm{P}^{M T}$ so after the deduction of the power required by the load, one of the options of using EGP is the charging of the 
battery. Despite this fact, as it is observed from Figure 7(b), the optimization algorithm decides to use EGP for feeding the loads including DR and EWH. In the EMS-MGSA algorithm, the selection of generation unit is included by considering the minimization of the objective function. MT in the MCEMS algorithm is off at $46 \%$ of the times while in the EMS-MGSA algorithm is around 14\% on during MG daily operation.

As it is observed from Figure 6(b), in the EMS-MGSA algorithm, MT is used more than ES for supplying the consumers. Although, $\lambda_{t}^{\prime M C P}$ in most of the time intervals is lower than $\lambda_{\mathrm{t}}^{\mathrm{MCP}}$.

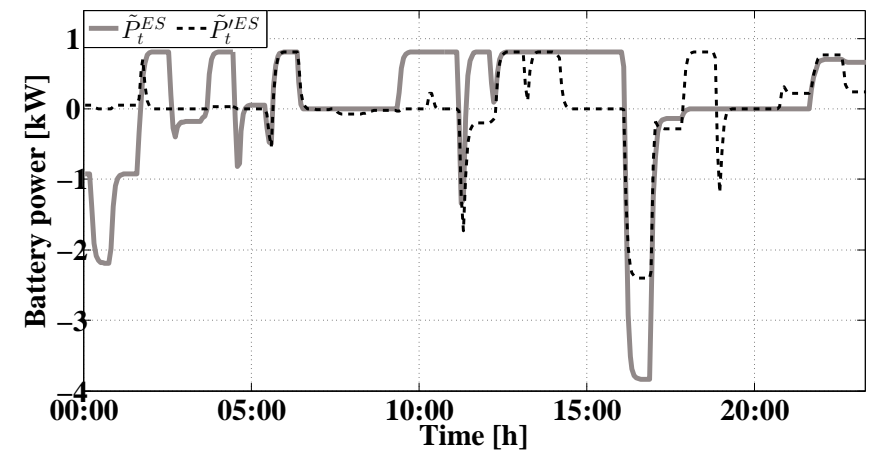

(a) ES

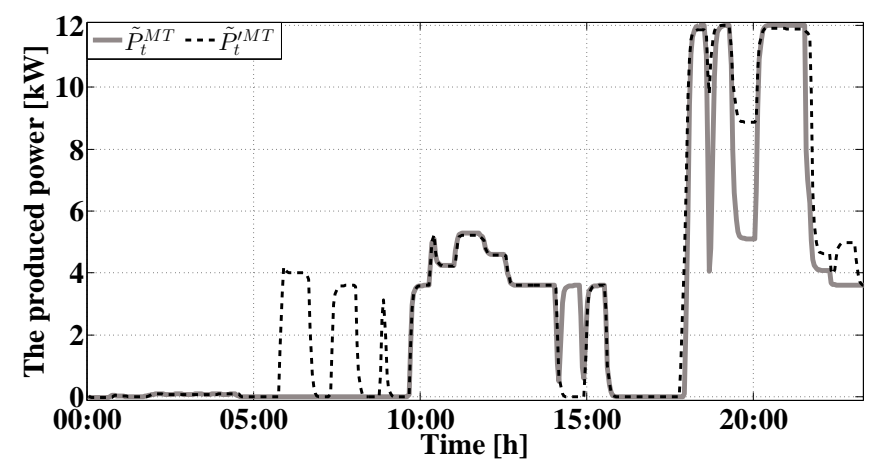

(b) MT

Figure 6: ES and MT power during system operation

The power consumed by DR, EWH, ES during charging and discharging, UP and EGP are shown as a bar graph in Figures. 7(a) and 7(b). ES is operating in charging mode around $42 \%$ of the times. ES in the MCEMS algorithm is generated $\overline{\mathrm{P}}^{\mathrm{ES}+}$ during $31 \%$ of the time operation. This percentage in the EMS-MGSA algorithm reaches $8.33 \%$ of the times. On the other hand, as it is observed from Figure 5, SOC in the EMS-MGSA algorithm is much better than the MCEMS algorithm. This fact shows that the algorithm based on optimization makes better decisions for using the EGP of the microsources regarding the best use of the electric power generation resources and storage devices in the MG.

MT is entered service with the power $\overline{\mathrm{P}}^{\mathrm{MT}}$ at the same time. From Figure 7(a), it is evident that most of DR is fed in the time interval 10:00 A.M to 15:00 P.M. As seen from Figure 7(b), all the DR in EMS-MGSA is fed during 00:00-
04:30 periods. In this time period, $\lambda_{t}^{\prime M C P}$ is variable between $0.16 € / \mathrm{kWh}$ to $0.4 € / \mathrm{kWh}$. Hence, significant reduction in the cost to supply DR is done by the optimization algorithm. In the time intervals that the scenarios are occurred (time interval 17:00-21:00) and the consumed load is decreased (the system is encountered UP), average of $\lambda_{t}^{M C P}$ is equal to $1.2 € / \mathrm{kWh}$. While in this time interval, the average value of $\lambda_{t}^{\prime M C P}$ is equal to $1 € / \mathrm{kWh}$. It means that the penalty cost is substantially reduced in the optimization algorithm. So, by feeding DR in the first 6 hours of system operation, the optimization algorithm presents the best choice for its feeding with the least possible expense. EWH in the MCEMS algorithm is only fed in the time interval 15:00-16:30. In this time interval, the average of $\lambda_{t}^{M C P}$ is equal to $0.51 € / \mathrm{kWh}$.

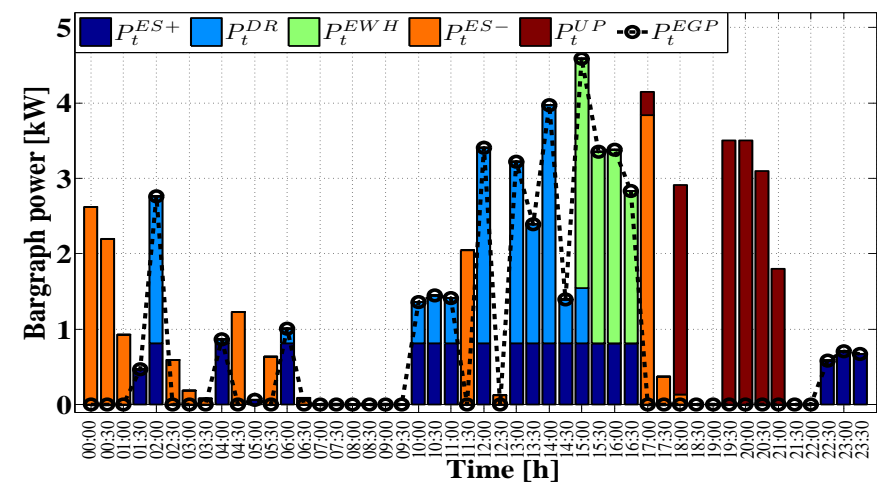

(a) MCEMS

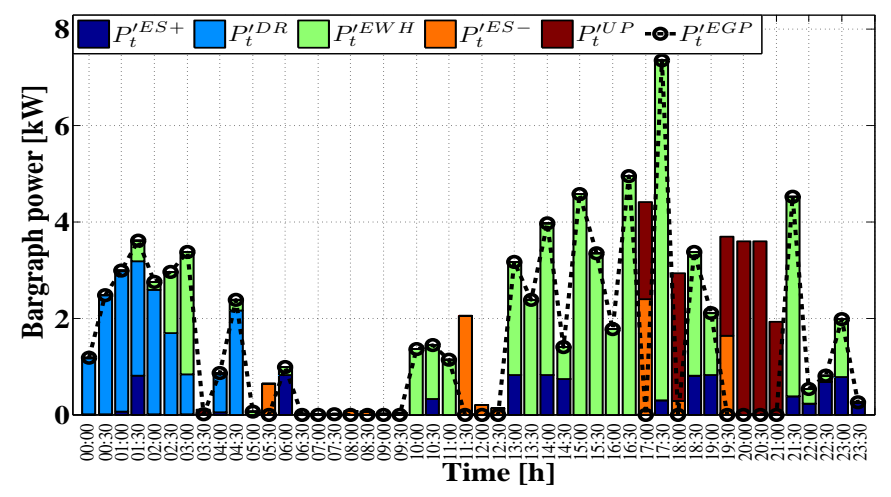

(b) EMS-MGSA

Figure 7: The bargraph related to the responsible loads power, ES discharging and UP during system performance

The proposed algorithm for solving EMS problem is implemented in MATLAB 8.01 platform and executed with i53320M CPU, 2.6 GHz desktop computer with 4GB RAM. In order to compare the computation time of the proposed algorithm, absolute CPU time is maintained in Table 2. It is also compared with reported simulation times in [20] to implement EMS based on particle swarm optimization (PSO) (EMS-PSO) algorithm. The obtained results shown that CPU can devote the less execution time (reduced by around 38\%) for the proposed algorithm compare to EMS-PSO algorithm. Apparently, the technical contribution of the proposed EMSMGSA algorithm not only gives a fine solution to minimize 
Table 2: Average computational time for case study corresponding to 100 iteration

\begin{tabular}{lll}
\hline \hline & MGSA & PSO \\
\hline Execution time (s) & 17.12 & 27.45 \\
\hline
\end{tabular}

the total generation cost for the unit commitment problem in MG application, but also a good compromise between computation time and precision.

Convergence curves of EMS-MGSA and EMS-PSO algorithms are also depicted in Figure 8. Both EMS-MGSA and EMS-PSO employ the same maximum iteration settings (is set to 100 iterations). It can be observed that the proposed algorithm not only provides better solution quality with minimum generation cost but also has faster convergence rate than EMS-PSO. The dominant convergence characteristic of the proposed EMS-MGSA is more obvious when applied to a large-scale system including multiple MGs with a lot of variables.

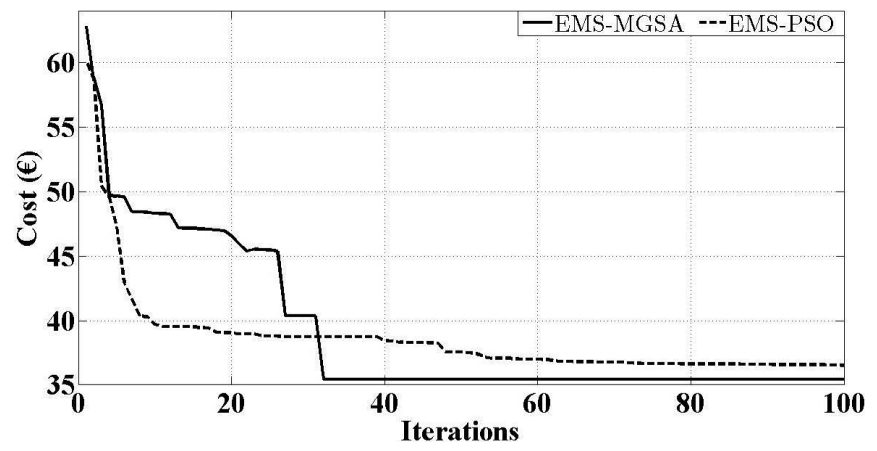

Figure 8: The cost convergence curve

MCP is shown in Figure 9 at each time interval. Its average value is also mentioned in each 6 hours period in Table 3 . At the first 6 hours of system operation, the average value of MCP in the EMS-MGSA algorithm is much less than the MCEMS algorithm. This means that feeding RLD loads (that is DR and EWH) at this time interval is the best option. As a result, by this way, less expenses will be paid for feeding them by consumers. At the second 6 hours of the system operation, despite the rising of $\lambda_{t}^{M C P}$, the EGP power is used for feeding ES and DR in the MCEMS algorithm. But as it is observed from Figure 7(a), most of the EGP power is used for feeding EWH which presents much less offer relative to ES and DR. In the third 6 hours of system operation, the average value of MCP is reduced in both of the algorithms. Considering MCEMS algorithm, in half of this period, EGP power is used only for feeding DR and ES. When feeding DR is completed, the rest of the time is used to feed ES and EWH, respectively. However, EGP in the EMS-MGSA algorithm is used for feeding EWH most of the times. At the last 6 hours of system operation, EGP power in the MCEMS algorithm is used only
Table 3: The average value of MCP in each 6 hour period of system performance

\begin{tabular}{|c|c|c|c|c|}
\hline 00:00-06:00 & $06: 00-12: 00$ & $12: 00-18: 00$ & $18: 00-24: 00$ & \\
\hline 0.62 & 0.49 & 0.56 & 0.41 & $\lambda_{\mathrm{t}}^{\text {MCP }}$ \\
\hline 0.60 & 0.33 & 0.34 & 0.31 & $\lambda_{\mathrm{t}}^{\text {MCP }}$ \\
\hline
\end{tabular}

for charging ES. Dispite the ES charge offer is higher than the DR and EWH offers and the average value of $\lambda_{t}^{M C P}$ is more than its average value in other periods, an adequate chosen is not intended for the consumers. However, EGP in the optimization algorithm is mostly allocated for feeding EWH that has the least offer among the consumers.

Both experimental and simulation results show that EMSMGSA algorithm is capable to operate in optimal scheduling, optimal operation, economic dispatch and demand side management in the best possible way. The total generation cost and MCP are reduced in the proposed algorithm by efficient management of generation, storage and load assets, by $18 \%$ in comparison with MCEMS.

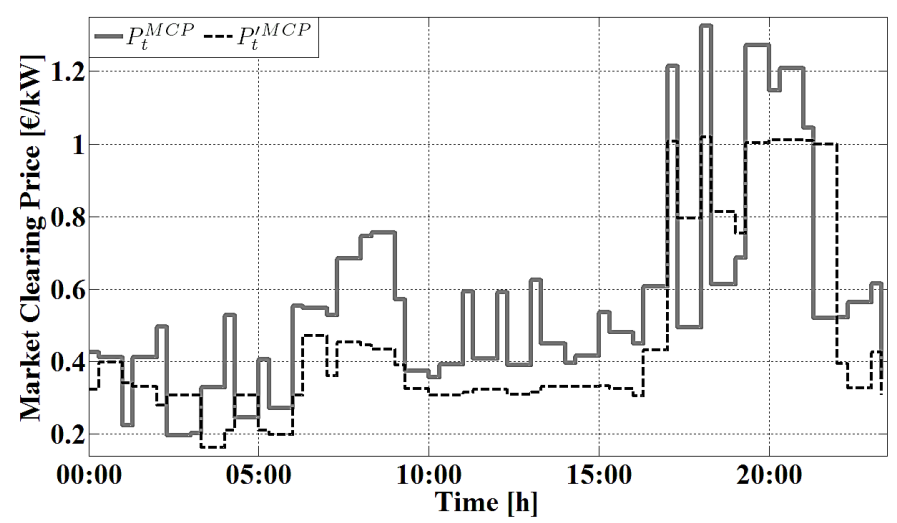

Figure 9: MCP for each interval during the system daily operation

\section{Conclusions}

The obtained results have demonstrated the effectiveness of EMS-MGSA algorithm in solving the optimal operation point within isolated MG. The optimal power setpoints for microsources has been achieved based on previous information and real-time experimental data by noting the fulfillment of all technical constraints. The optimization method in accordance with GSA approach has been introduced to minimize the production cost as well as to increase the system efficiency. A strategy for smart grid has been developed to shift the load and the accordance of power generation by renewable and non-renewable sources. This solution has been implemented experimentally over the IRECs' MG system. Its efficiency and performance has been verified by using different scenarios. The priority index for consumers to participate in LEM has been considered based on the offer by 
them and minimizing objective function. The obtained results have shown the improvement of the overall system operation in comparison with MCEMS. The experimental and simulation results have shown that the increase in the percentage of the load shifting not only could yield more flexibility to the system but also cause the use excess of generated energy. Moreover, it has been observed that the system efficiency in finding the best way would lead to maximize the usage of the power generated by renewable sources. In addition, consumers have participated in DR with high priority index could be supplied with less cost (approximately $18 \%)$. It is worth to remark that EMS-MGSA has operated better in reducing overall peak demand, the optimum operation of the present micro-sources and decreasing the total generation cost relative to the MCEMS algorithm. The proposed algorithm proves the efficiency of GSA method for managing and exchanging power in smart grids. Eventually, using the proposed algorithm will enable utility companies to have an energy management tool with the optimization ability of using non-dispatchable and ES assets to supply industrial/commercial and household loads.

\section{Acknowledgments}

The research leading to these results has received funding from the European Union seventh framework program "FP7SMARTCITIES-2013” under grant aggreement 608860.

\section{References}

[1] M. Marzband, A. Sumper, A. Ruiz-Álvarez, J. L. Domínguez-García, B. Tomoiagă, "experimental evaluation of a real time energy management system for stand-alone microgrids in day-ahead markets", Applied Energy 106 (0) (2013) 365-76.

[2] A. Parisio, E. Rikos, G. Tzamalis, L. Glielmo, "use of model predictive control for experimental microgrid optimization", Applied Energy, 115 (0) (2014) 37-46.

[3] B. Zhao, X. Zhang, P. Li, K. Wang, M. Xue, C. Wang, "optimal sizing, operating strategy and operational experience of a stand-alone microgrid on dongfushan island", Applied Energy, 113 (0) (2014) 1656-66.

[4] G. Kyriakarakos, D. D. Piromalis, A. I. Dounis, K. G. Arvanitis, G. Papadakis, "intelligent demand side energy management system for autonomous polygeneration microgrids", Applied Energy, 103 (0) (2013) 39-51.

[5] W. Su, A. Q. Huang, "a game theoretic framework for a next-generation retail electricity market with high penetration of distributed residential electricity suppliers", Applied Energy, 119 (0) (2014) 341-50.

[6] Y. H. Chen, S. Y. Lu, Y. R. Chang, T. T. Lee, M. C. Hu, "economic analysis and optimal energy management models for microgrid systems: A case study in taiwan", Applied Energy, 103 (0) (2013) 145-54.

[7] B. Wang, M. Sechilariu, F. Locment, "intelligent DC microgrid with smart grid communications: Control strategy consideration and design", IEEE Transactions on Smart Grid, 3 (4) (2012) 2148-56.

[8] S. A. Pourmousavi, M. H. Nehrir, "real-time central demand response for primary frequency regulation in microgrids", IEEE Transactions on Smart Grid, 3 (4) (2012) 1988-96.

[9] R. P. Behnke, C. Benavides, F. Lanas, B. Severino, L. Reyes, J. Llanos, D. Saez, "a microgrid energy management system based on the rolling horizon strategy", IEEE Transactions on Smart Grid 4 (2) (2013) 9961006.

[10] M. F. Ghazvini, H. Morais, Z. Vale, "coordination between mid-term maintenance outage decisions and short-term security-constrained scheduling in smart distribution systems", Applied Energy, 96 (0) (2012) 281-91.
[11] T. Logenthiran, D. Srinivasan, A. M. Khambadkone, H. N. Aung, "multiagent system for real-time operation of a microgrid in real-time digital simulator", IEEE Transactions on Smart Grid, 3 (2) (2012) 925-33.

[12] S. Shao, M. Pipattanasomporn, S. Rahman, "grid integration of electric vehicles and demand response with customer choice", IEEE Transactions on Smart Grid, 3 (1) (2012) 543-50.

[13] W. W. Weaver, P. T. Krein, "game-theoretic control of small-scale power systems", IEEE Transactions on Power Delivery, 24 (3) (2009) 1560-67.

[14] B. Daemi, A. Abdollahi, B. Amini, F. Matinfar, "digitally-signed distribution power lines: A solution which makes distribution grid intelligent", IEEE Transactions on Power Delivery, 25 (3) (2010) 1434-39.

[15] J. A. Martinez, F. de Leon, A. Mehrizi-Sani, M. H. Nehrir, C. Wang, V. Dinavahi, "tools for analysis and design of distributed resourcespart II: Tools for planning, analysis and design of distribution networks with distributed resources", IEEE Transactions on Power Delivery, 26 (3) (2011) 1653-662.

[16] N. Padhy, "unit commitment-a bibliographical survey", IEEE Transactions on Power Systems, 19 (2) (2004) 1196-205.

[17] Y. Y. Hong, M. C. Hsiao, Y. R. Chang, Y. D. Lee, H. C. Huang, "multiscenario underfrequency load shedding in a microgrid consisting of intermittent renewables", IEEE Transactions on Power Delivery, 28 (3) (2013) 1610-17.

[18] C. Chen, S. Duan, T. Cai, B. Liu, G. Hu, "smart energy management system for optimal microgrid economic operation", IET Renewable Power Generation, 5 (3) (2011) 258-67.

[19] F. Zhuang, F. D. Galiana, "unit commitment by simulated annealing", IEEE Transactions on Power Systems, 5 (1) (1990) 311-18.

[20] M. Marzband, Experimental validation of optimal real-time energy management system for microgrids, $\mathrm{PhD}$ thesis, Departament d'Enginyeria Elèctrica, EU d'Enginyeria Tècnica Industrial de Barcelona, Universitat Politècnica de Catalunya (2013).

[21] M. A. Hassan, M. A. Abido, "optimal design of microgrids in autonomous and grid-connected modes using particle swarm optimization", IEEE Transactions on Power Electronics, 26 (3) (2011) 755-69.

[22] A. K. Basu, S. Chowdhury, S. P. Chowdhury, "impact of strategic deployment of CHP-based DERs on microgrid reliability", IEEE Transactions on Power Delivery 25 (3) (2010) 1697-705.

[23] L. Wang, Z. Wang, R. Yang, "intelligent multiagent control system for energy and comfort management in smart and sustainable buildings", IEEE Transactions on Smart Grid 3 (2) (2012) 605-17.

[24] T. Niknam, F. Golestaneh, A. Malekpour, "probabilistic energy and operation management of a Microgrid containing Wind-PhotovoltaicFuel cell generation and energy storage devices based on point estimate method and self-adaptive gravitational search algorithm", Energy 43 (1) (2012) 427-37.

[25] B. Shaw, V. Mukherjee, S. Ghoshal, A novel opposition-based gravitational search algorithm for combined economic and emission dispatch problems of power systems, International Journal of Electrical Power \& Energy Systems 35 (1) (2012) 21-33.

[26] S. Mondal, A. Bhattacharya, S. Dey, Multi-objective economic emission load dispatch solution using gravitational search algorithm and considering wind power penetration, International Journal of Electrical Power \& Energy Systems 44 (1) (2013) 282-92.

[27] S. Jiang, Z. Ji, Y. Shen, A novel hybrid particle swarm optimization and gravitational search algorithm for solving economic emission load dispatch problems with various practical constraints, International Journal of Electrical Power \& Energy Systems 55 (0) (2014) 628-44.

[28] M. Marzband, A. Sumper, J. L. Domínguez-García, R. Gumara-Ferret, "experimental validation of a real time energy management system for microgrids in islanded mode using a local day-ahead electricity market and MINLP", Energy Conversion and Management 76 (0) (2013) 314 22.

[29] H. Yamin, Q. El-Dwairi, S. Shahidehpour, "a new approach for gencos profit based unit commitment in day-ahead competitive electricity markets considering reserve uncertainty", International Journal of Electrical Power \& Energy Systems, 29 (8) (2007) 609-16.

[30] M. Marzband, A. Sumper, M. Chindriş, B. Tomoiagă, "energy management system of hybrid microgrid with energy storage", The International Word Energy System Conference (WESC), Suceava, Romania, 2012.

[31] M. Marzband, A. Sumper, "implementation of an optimal energy man- 
agement within islanded microgrid", International Conference on Renewable Energies and Power Quality (ICREPQ), Cordoba, Spain, 2014.

[32] E. Rashedi, H. Nezamabadi-pour, S. Saryazdi, "GSA: A gravitational search algorithm", Information Sciences 179 (0) (2013) 2232-48.

[33] M. A. Behrang, E. Assareh, M. Ghalambaz, M. Assari, A. R. Noghrehabadi, "forecasting future oil demand in iran using GSA (gravitational search algorithm)", Energy 36 (9) (2011) 5649-54.

[34] S. Duman, U. Güvenç, Y. Sönmes, N. Yörükerenc, "optimal power flow using gravitational search algorithm", Energy Conversion and Management 59 (0) (2012) 86-95.

[35] A. Ruiz-Álvarez, A. Colet-Subirachs, F. A.-C. Figuerola, O. GomisBellmunt, A. Sudriá-Andreu, "operation of a utility connected microgrid using an IEC 61850-based multi-level management system", IEEE Transactions on Smart Grid 3 (2) (2012) 858-65.

[36] A. Colet-Subirachs, A. Ruiz-Álvarez, O. Gomis-Bellmunt, F. ÁlvarezCuevas-Figuerola, A. Sudriá-Andreu, "centralized and distributed active and reactive power control of a utility connected microgrid using IEC 61850", IEEE Systems Journal 6 (1) (2012) 58-67. 


\section{List of Tables}

1 The supply bids by the generation and consumers assets $[€ / \mathrm{kWh}] \ldots \ldots \ldots \ldots \ldots \ldots \ldots$

2 Average computational time for case study corresponding to 100 iteration . . . . . . . . . . . . . . 10

3 The average value of MCP in each 6 hour period of system performance . . . . . . . . . . . . . . . 10 